\title{
POTENTIALITY OF USING DIGITAL WAVELET/QMF PYRAMIDS IN REMOTELY SENSED SATELLITES' IMAGES CLASSIFICATION
}

\author{
Ahmed SERWA $10 *$ \\ Faculty of Engineering in El-Mataria, Helwan University, Cairo, Egypt
}

Received 25 October 2019; accepted 05 December 2020

\begin{abstract}
Wavelet or quadrature mirror filter (QMF) satellites' images are not commonly used in classification because of the modification in spectral responses that may confuse any classifier. Boundary pixels are hardly classified correctly in pixel-based classification especially in medium and coarse resolution. In such case, the sudden change in landcover is not measurable by the classifiers because the pixel may contain mor than one class. This research work is a trial to investigate the proper enhancement in accuracy that may occur by using wavelet/QMF bands' pyramids are in classification instead of the original image bands. The reference map is prepared traditionally to measure the performance of the new system. The Wavelet/QMF image is constructed for each band of the satellite image. Then the classification is carried out for both the Wavelet/QMF image pyramid and the original satellite image using competitive learning neural networks (CLNN) method. The evaluation is carried out by comparing the classified Wavelet/QMF image with the classified original image. A statistical test is carried out to study the significance of using the classified Wavelet/QMF image in classification.
\end{abstract}

Keywords: remote sensing, classification, Wavelet/QMF pyramid, accuracy assessment.

\section{Introduction}

Cost and time reduction can be achieved in remote sensing systems by automation (Serwa, 2016). Digital image pyramids is expressed as an abstraction tools that can increase automation accompanied by a change in size, resolution and grey levels (Serwa, 2020b). The problem of changing the original spectral reflectance of the objects in remote sensing images lead excluding the use of processed images in classification (Serwa, 2020b). On contrary, some researchers tended to explore the effect of using digital image pyramids in remote sensing classification such as Serwa (2020b) who made a complete investigation of using Gaussian pyramids in classification. While Yang et al. (2018) made a ships detection using multi scaled feature pyramids. Serwa (2012) made a compression for MS satellites' images producing a single band pyramid produced for remote sensing classification without the to use the full bands images. Zhang and Yang (2013) made an analysis in the frequency domain by area of interest extraction and salient region detection for satellites' images. Yue et al. (2016) apply spatial pyramid pooling using deep learning framework for hyperspectral image classification. While pre-trained Alex-Net architecture having pyramid pooling and supervision were used by Han et al. (2017) for high spatial resolution remote sensing classification. According to the above literature review it can be deducted that using Wavelet/QMF pyramids of remote sensing images in remote sensing classification is rarely handled. Accordingly, enhancement of classification by using Wavelet/QMF pyramid can be investigated. In this research the classification accuracy is used as measurement of performance of using Wavelet/QMF pyramid in classification.

\section{Digital pyramids}

There are four common types of pyramids: Gaussian, Laplacian, wavelet and steerable pyramids (according to (Erdem, n.d.)). Each pyramid type has its own attributes concerning with structure, volume and purpose. Mostly, each pyramid represents a scalar abstraction from the original pyramid (level $J$ ) as shown in Figure 1. The minimum obtainable pyramid size is $1 \times 1$ pyramid (level 0 ) which expresses the single pixel image. Every pyramid may be used in such application according to its attributes and size. Some applications require up sampled pyramid and others require down sampled of the original image (Serwa, 2020a). The common normal form of the digital pyramid

${ }^{\star}$ Corresponding author. E-mail: ahmed_serwa@yahoo.com 
is shown in Figure 2. The number of reforming pixels can be computed according to (Gonzalez et al., 2009) as:

$$
N^{2}\left(1+\frac{1}{4^{1}}+\frac{1}{4^{2}}+\ldots \frac{1}{4^{P}}\right) \leq\left(\frac{4}{3}\right) N^{2},
$$

where $N$ is the original image dimension in pixels and $P$ is an integer number that determine the pyramid rank $(J-P)$ the pyramid rank. Figure 3 indicates the common image pyramids: Gaussian, Laplacian, wavelet and steerable pyramid.

On can deduct that Gaussian pyramid is blurred and subsampled form of the original image and it adds scale invariance to fixed-size image. The most common blurring filter used mostly is the mean filter. Laplacian pyramid shows the information added in Gaussian pyramid at each spatial scale and it can be used for noise minimization. Wavelet/QMF pyramid is sub band passed form with aliasing with non-oriented sub bands. It can be used for image enhancement. Steerable pyramid shows components at each size and orientation separately with no aliased sub bands. It is good for texture and feature analysis. But it is overcomplete and it has a high frequency residual form. In this research Wavelet/QMF pyramid with average filter is used due to it is the most used in most of remote sensing applications (Serwa, 2020b).

\section{Methodology}

The research is an investigation for the classification accuracy when using the full featured Wavelet/QMF pyramids in multispectral image classification. Landsat $8 \mathrm{MS}$ image is used as an application. The reference of the study area is built in traditional way pixel by pixel to guarantee the reliable performance. All the main tasks such as: spatial sub setting, preparing regions of interest (ROIs), geo-referencing and corrections were done using ENVI software.

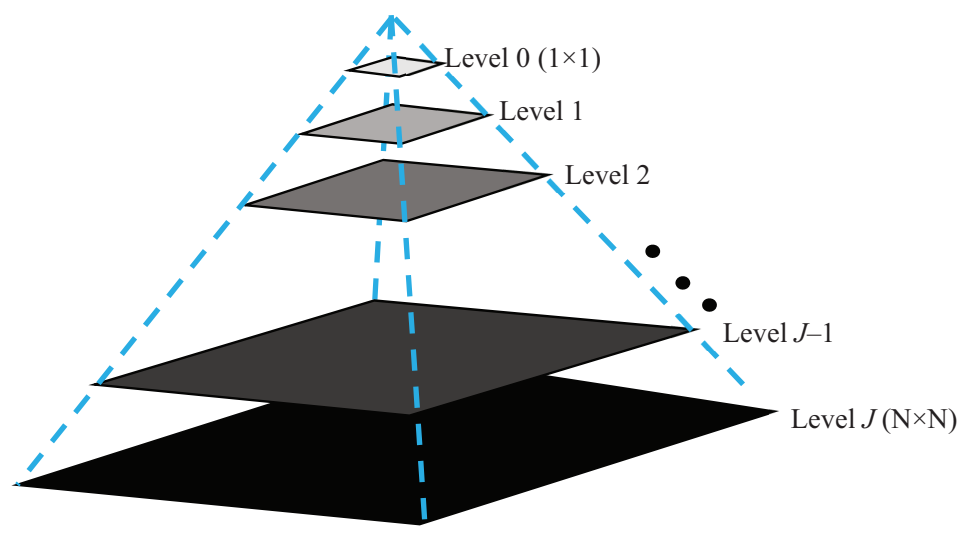

Figure 1. Digital pyramid form after (Serwa, 2020b)

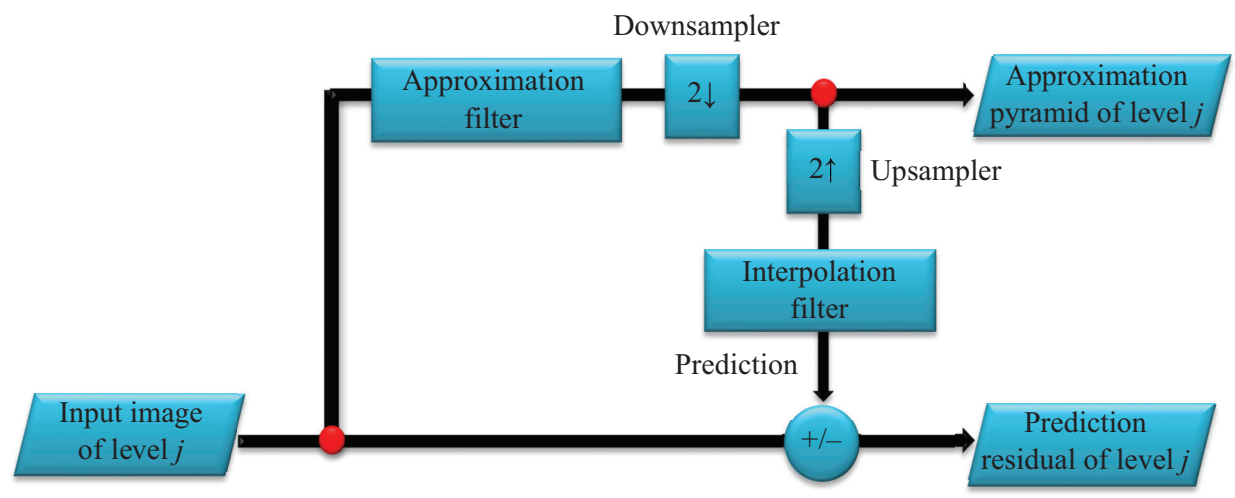

Figure 2. General construction process of digital pyramid (Serwa, 2020b)

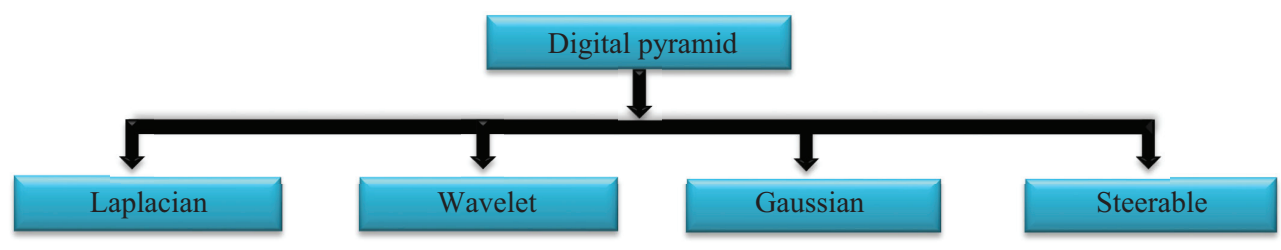

Figure 3. Common digital pyramids (Serwa, 2020b) 
Construction of full featured Wavelet/QMF pyramid for each band of the satellite image bands is carried out using ADIPRS (Advanced Digital Image Processor for Remote Sensing) by Serwa (2009). The general classification approach described in Serwa and El-Semary (2020) is applied because it is well stabilized and dependable. This research is different from Serwa (2020b) which is a processing in the image domain while this is a processing in the frequency domain.

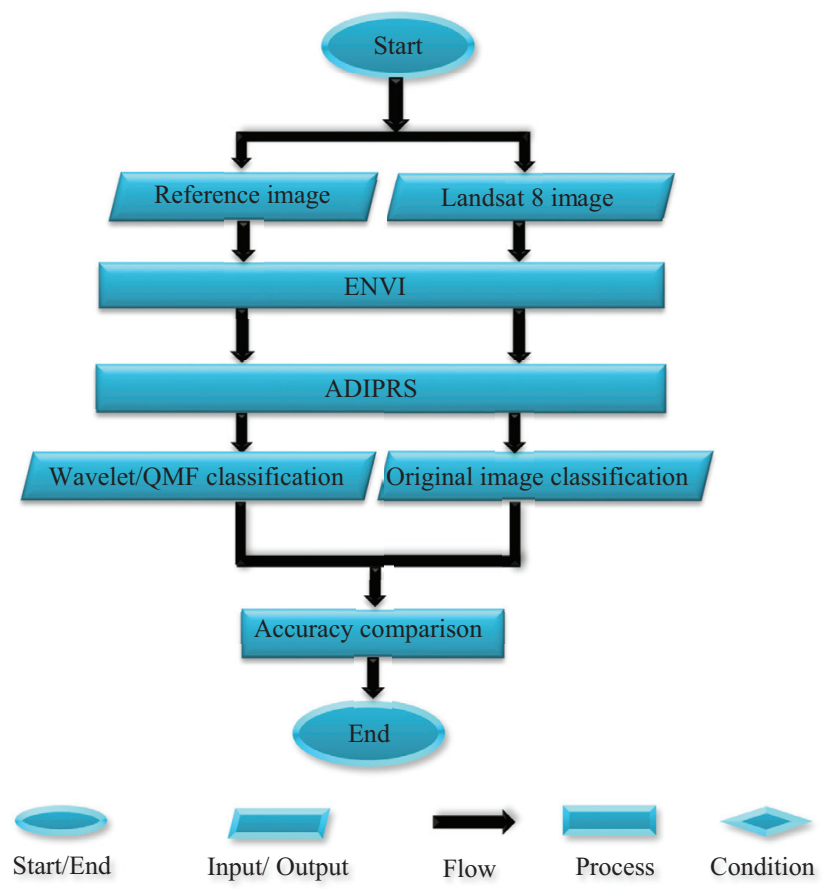

Figure 4. Block diagram of the research work

\subsection{System overview}

System overview can be abstracted using a flowchart diagram in Figure 4 . The system begins with input of both the original satellite image bands and the reference. ENVI software is used to construct the main tasks such as: spatial sub setting, preparing regions of interest and georeferencing. While ADIPRS software is used to establish the full featured Wavelet/QMF pyramid and to adopt the classification for both the original image and the Wavelet/QMF image. The reference is used to measure the accuracy of the classification of both images (original and Wavelet/QMF). Unsupervised algorithm of competitive learning neural networks (CLNN) is used in this research because the reference was prepared using it previously.

Both classified original and classified Wavelet/QMF images are compared with the reference to examine the accuracy. A comparison between the accuracy of the classification of the original image and the accuracy of the classification of the Wavelet/QMF image is done. A statistical test is carried out to investigate the significance of using Wavelet/QMF pyramid in remote sensing classification.

\subsection{Research data}

Landsat 8 satellite image is used in this study which covers a part of Port Said city in Egypt with an area of $600 \times 600$ pixels $\left(324 \mathrm{~km}^{2}\right)$. The reference map is constructed normally by AutoCAD maps and suite visits (with GPSMAP device). The study area on ENVI environment is shown in Figure 5 before the subsetting.

Figure 6 shows the reference to obtain the best testing performance. One must note that each band of the

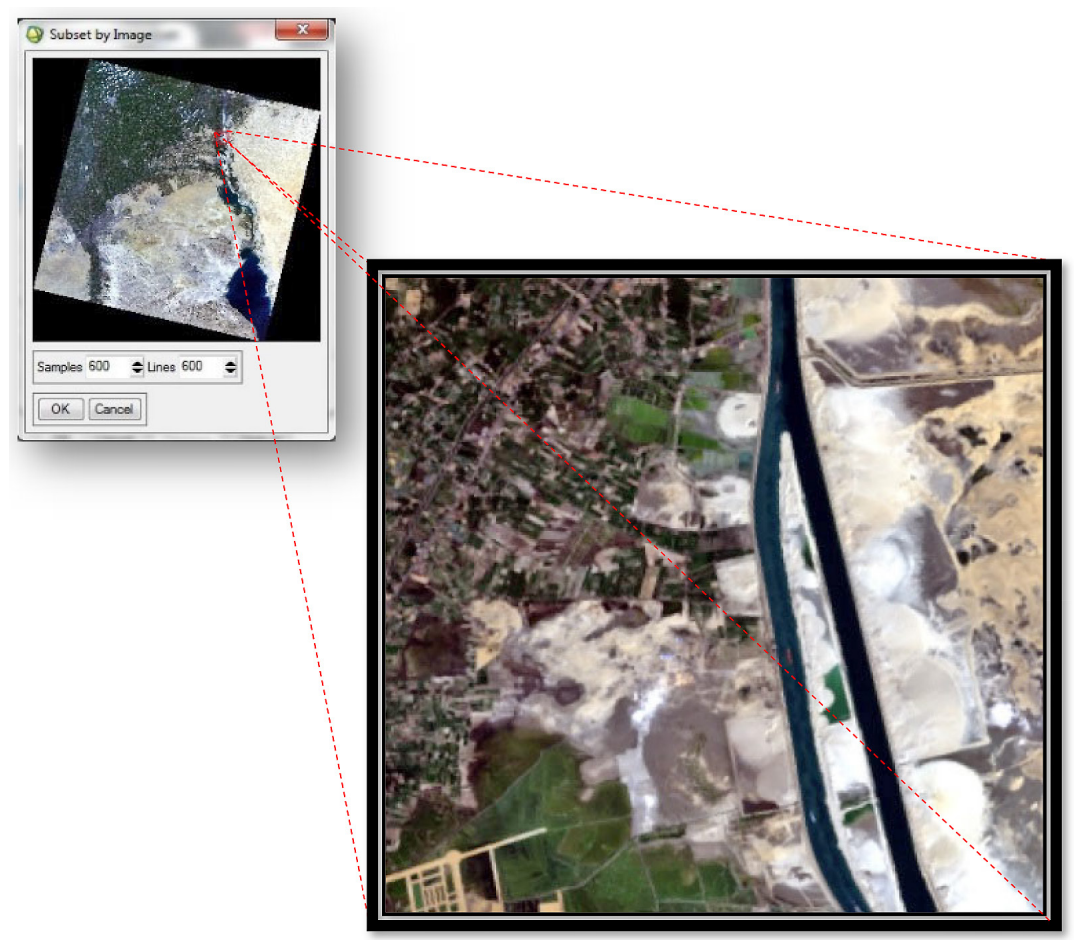

Figure 5. True color Landsat 8 for the study area (Serwa, 2020b) 


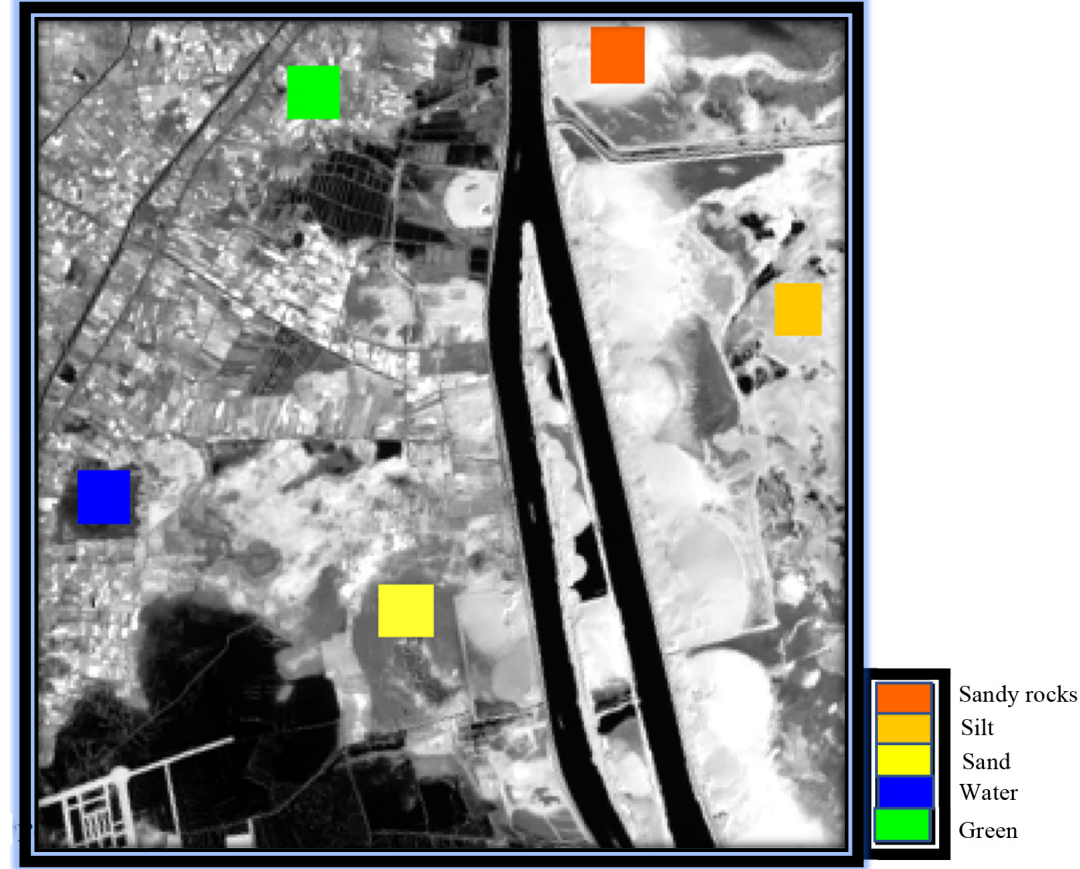

Figure 6. Reference for the study area

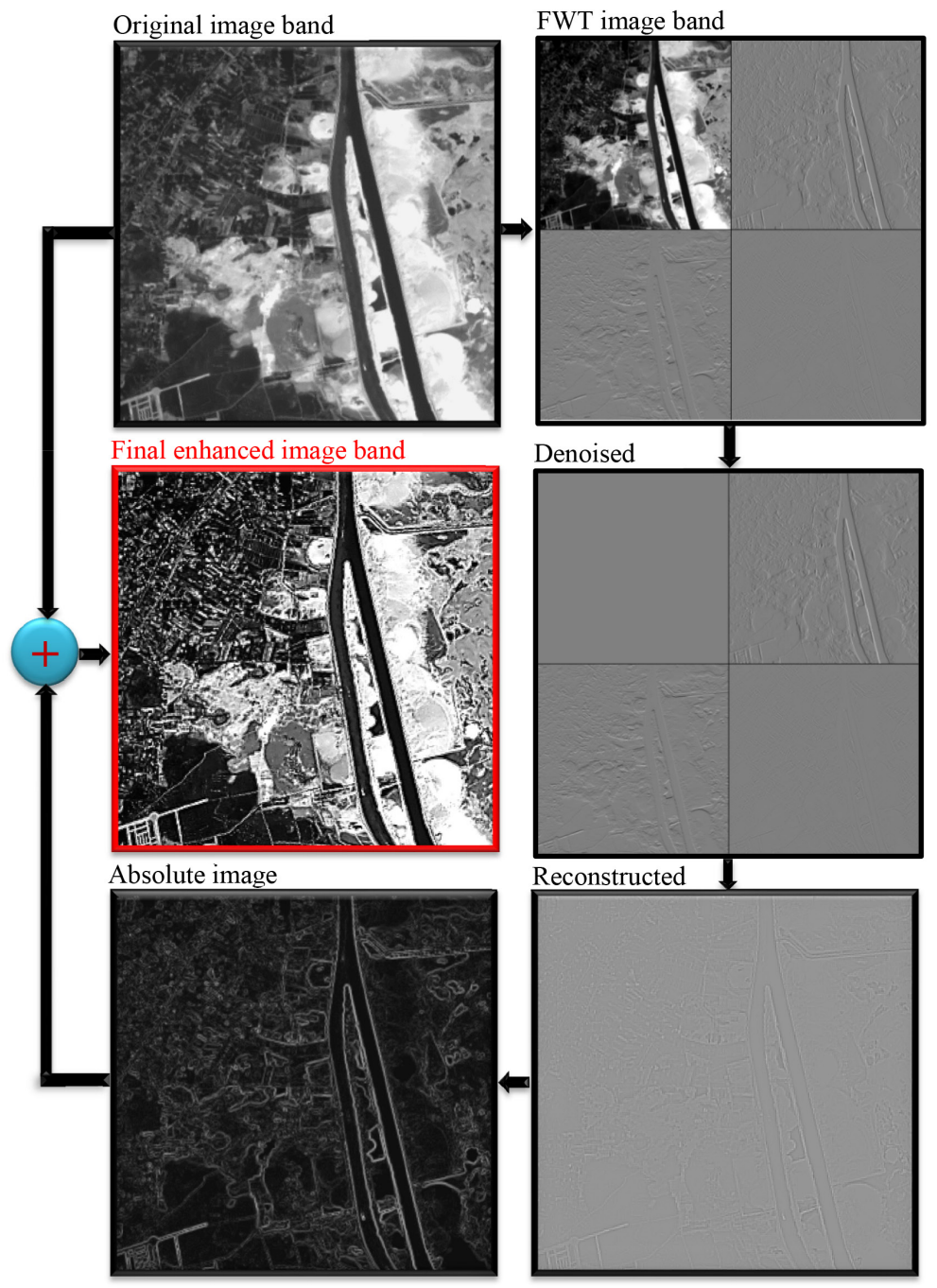

Figure 7. Construction of full featured Wavelet/QMF pyramid band 
satellite image is used separately to produce its Wavelet/QMF pyramid and so the MS Wavelet/QMF pyramid is a composition of seven pyramid bands of the original image. The satellite image is corrected from all necessary errors such as atmospheric, radiometric and geometric corrections. Precautions were taken such as selecting the area with off-cloud and off-relief (no remarkable levels variation).

\subsection{Processing}

Figure 7 indicates the steps of construction of full featured Wavelet/QMF pyramid of single band of the MS image. Construction of full featured Wavelet/QMF pyramid band can be obtained in steps. The first step is establishing the fast wavelet transformed (FWT) image. A noise removal must be done to produces the denoised form. Then the reconstructed version can be obtained from the denoised form.

The absolute band can be obtained by subtracting the gray color from the denoised image. The last step is adding the absolute image to the original image band to finally obtain the full featured Wavelet/QMF image band. The previous steps are carried out for all 8 bands of the MS image. ADIPRS software is used to construct the full featured Wavelet/QMF pyramid. CLNN classification in its unsupervised form (clustering) is used with overfitting then make classless merging to obtain the required classes.
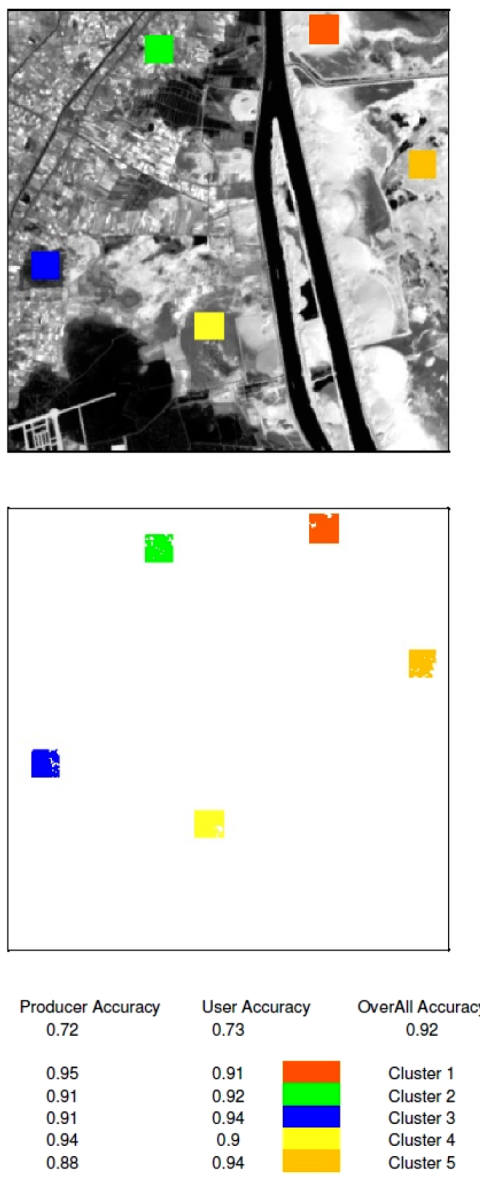

Figure 9. Classification report of the original image

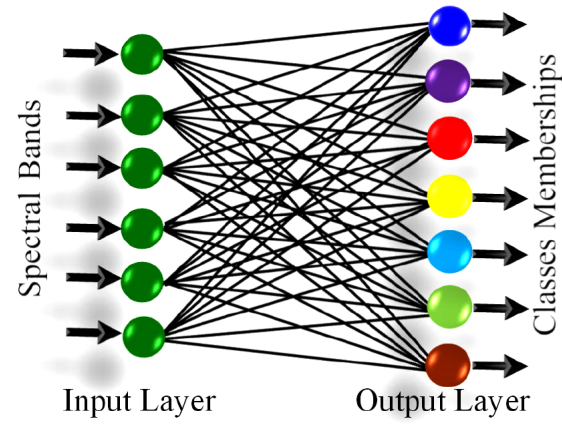

Figure 8. CLNN architecture

CLNN architecture is consisting of only input layer and output layer (without hidden layers) as shown in Figure 8. For more information about CLNN and its details see Serwa et al. (2010).

\section{Results and analysis}

The classification overall accuracy of $92 \%$ is obtained for the original image as shown in Figure 9. One must note the value of K-hat statistic (Kappa value) equals $90 \%$ and it is near the overall accuracy which means that the result is reliable. While Figure 10 shows the classification report of the Wavelet/QMF pyramid with overall accuracy of $93 \%$ and the value $\mathrm{K}$-hat statistic is $92 \%$. The last
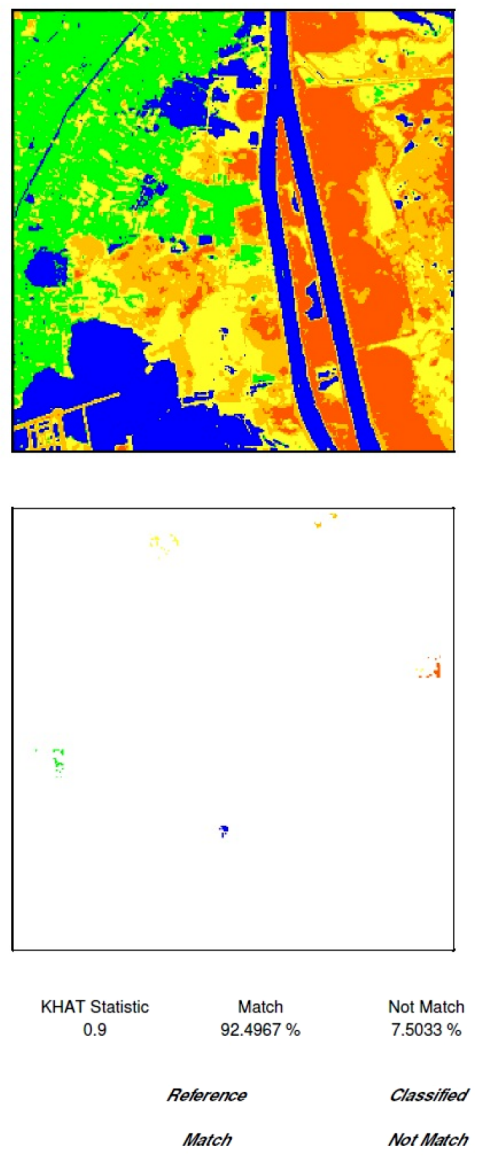

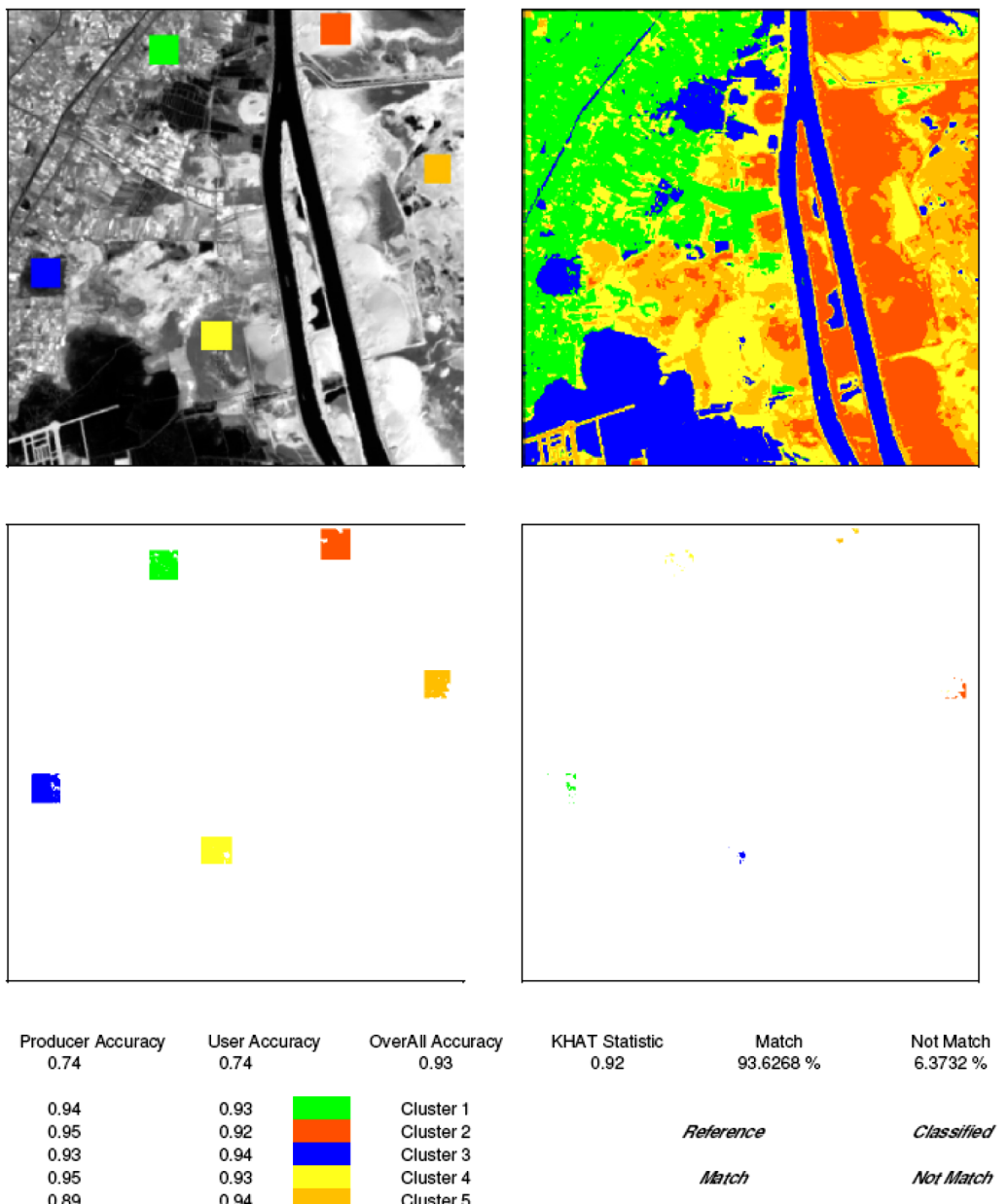

Figure 10. Classification report of the Wavelet/QMF pyramid

results indicate an enhancement in classification due to the narrower gab between the overall accuracy and the value of K-hat statistic. One must search for a reason for the enhancement in classification accuracy when using the Wavelet/QMF pyramid. The reason can be explained as most of miss classified pixels are boundary pixels. So, the full featured Wavelet/QMF pyramid decreases the miss classification in the boundary pixels.

A comparison between the classification accuracy of the original classified image and the Wavelet/QMF classified pyramid must be carried out. The enhancement in accuracy is $1 \%$ which means initially that using the full featured wavelet/QMF pyramid is better. A statistical test is adopted to make sure this idea. Traditionally, 95\% level of confidence can be assumed; so, the first hypothesis is that there is no significant difference between the two classification results while the alternative on is that there is a significant difference between them. The testing sample is $600 \times 600$ pixels $>30$ pixels so that degree of freedom for is $\infty$ for both hypotheses. By comparing the two overall accuracies' percentages one can use Ftest to evaluate the difference between them. The critical values can be retrieved from standard statistical tables showing the following:

$$
\begin{aligned}
& F_{\text {Calculated }}=(0.93 / 0.92)=1.011 ; \\
& F_{\text {Critical }}=1.0(\text { for degree of freedom } \infty \text { for S1 and S2). }
\end{aligned}
$$

So:

$F_{\text {Calculated }}>F_{\text {Critical }}$ tables so the difference is significant.

We must accept the hypothesis of there is a significant difference between the two results and reject the first one.

\section{Conclusions}

According to the research objective, using Wavelet/QMF image pyramid somewhat enhance the classification accuracy. The reason of the enhancement in classification accuracy can be explained by increasing the performance of the classifier concerning with the boundary pixel by making a true decision for. The full featured Wavelet/QMF pyramid solved the problem of the boundary pixels by make a sharp change in the spectral properties. The classifier reduces the confusion when examining the boundary pixels in the Wavelet/QMF pyramid. The research objective is achieved by indicating some unknown points 
concerning with using the Wavelet/QMF pyramid in remote sensing classification.

\section{References}

Erdem, E. (n.d.). BBM 413: fundamentals of image processing. https://web.cs.hacettepe.edu.tr/ erkut/bbm413.f16/index. html

Gonzalez, R. C., Woods, R. E., \& Masters, B. R. (2009). Digital image processing, third edition. Journal of Biomedical Optics, 14(2), 029901. https://doi.org/10.1117/1.3115362

Han, X., Zhong, Y., Cao, L., \& Zhang, L. (2017). Pre-trained alexnet architecture with pyramid pooling and supervision for high spatial resolution remote sensing image scene classification. Remote Sensing, 9(8), 848. https://doi.org/10.3390/rs9080848

Serwa, A. (2009). Automatic extraction of topographic features from digital images [PhD thesis]. Azhar University.

Serwa, A. (2012). New method for feature reduction of mss satellite bands to produce single equivalent band. Al-Azhar University Engineering Journal, 7(1), 519-526.

Serwa, A. (2016). Development of soft computational simulator for aerial imagery project planning. Surveying and Land Information Science, 75(2), 65-75.

Serwa, A. (2020a). Correction to: studying the potentiality of using digital gaussian pyramids in multi-spectral satellites images classification. Journal of the Indian Society of Remote Sensing. https://doi.org/10.1007/s12524-020-01210-8
Serwa, A. (2020b). Studying the potentiality of using digital gaussian pyramids in multi-spectral satellites images classification. Journal of the Indian Society of Remote Sensing. https://doi.org/10.1007/s12524-020-01210-8

Serwa, A., Ali, M. E.-N. O., \& Dief-Allah, M. A. M. (2010). Potential of fusion of fuzzy based and neural network classifiers for unsupervised classification. Al-Azhar University Engineering Journal, 5(1), 713-726.

Serwa, A., \& El-Semary, H. H. (2020). Semi-automatic general approach to achieve the practical number of clusters for classification of remote sensing MS satellite images. Spatial Information Research, 28, 203-213. https://doi.org/10.1007/s41324-019-00283-Z

Yang, X., Sun, H., Fu, K., Yang, J., Sun, X., Yan, M., \& Guo, Z. (2018). Automatic ship detection in remote sensing images from google earth of complex scenes based on multiscale rotation dense feature pyramid networks. Remote Sensing, 10(1), 132. https://doi.org/10.3390/rs10010132

Yue, J., Mao, S., \& Li, M. (2016). A deep learning framework for hyperspectral image classification using spatial pyramid pooling. Remote Sensing Letters, 7(9), 875-884. https://doi.org/10.1080/2150704X.2016.1193793

Zhang, L., \& Yang, K. (2013). Region-of-interest extraction based on frequency domain analysis and salient region detection for remote sensing image. IEEE Geoscience and Remote Sensing Letters, 11(5), 916-920.

https://doi.org/10.1109/LGRS.2013.2281827 